\title{
Multilevel lumbar spine infection due to poor dentition in an immunocompetent adult: a case report
}

\author{
Michaela B. Quast ${ }^{1}$, Carrie M. Carr ${ }^{2}$ and W. Michael Hooten ${ }^{3^{*}}$
}

\begin{abstract}
Background: Although spinal infections have been reported following dental procedures, development of a spinal infection attributed to poor dentition without a history of a dental procedure in an immunocompetent adult has not been previously reported. Here we provide a case report of a multilevel lumbar spine infection that developed in an immunocompetent adult with poor dentition.

Case presentation: A 63-year-old white male man with past medical history of hypertension presented to a hospital emergency department with a 4-month history of progressively worsening low back pain. A musculoskeletal examination demonstrated diffuse tenderness in his lumbar spine area and the results of a neurological examination were within normal limits. Computed tomography and magnetic resonance imaging of his lumbar spine demonstrated a prevertebral and presacral fluid collection ventral to the L4 to L5 and L5 to S1 interspaces. Blood cultures grew pan-sensitive Streptococcus intermedius in four of four bottles within 45 hours. Using computed tomography guidance, three core biopsies of the $L 4$ to $L 5$ interspace were taken and subsequent cultures were positive for Streptococcus intermedius. He reported that his last episode of dental care occurred more than 20 years ago and a dental panoramic radiograph demonstrated significant necrotic dentition. Ten teeth were extracted and the necrotic dentition was assumed to be the most likely source of infection. On hospital dismissal, he received a 12-week course of intravenously administered ceftriaxone followed by an 8-week course of orally administered cefadroxil pending repeat imaging.

Conclusions: This case report demonstrates the importance of determining the source of infection in a patient with a spontaneous spinal infection. Even in the absence of a recent dental procedure, dentition should be considered a possible source of infection in an immunocompetent patient who presents with a spontaneous spinal infection.
\end{abstract}

Keywords: Lumbar spine, Epidural abscess, Dentition, Immunocompetent

\section{Background}

Nonsurgery-related spinal infections represent an uncommon but devastating group of infectious diseases. The incidence of spontaneous epidural abscess in a geographically defined population was reported to be 0.88 cases per 100,000 person-years (95\% confidence interval, 0.27 to 1.48) [1]. This incidence was comparable to the incidence of spontaneous epidural abscess among hospitalized patients which was reported to be 0.33 to 1.96 abscesses per 10,000 hospital admissions per year [2].

\footnotetext{
*Correspondence: hooten.william@mayo.edu

${ }^{3}$ Department of Anesthesiology, Mayo Clinic College of Medicine, 200 First St SW, Rochester, MN 55905, USA

Full list of author information is available at the end of the article
}

Following use of an epidural catheter for operative anesthesia or postoperative analgesia, the odds of developing an epidural abscess has been reported to range from 0 to 1:1930 [3, 4]. Various spinal infections including lumbar and cervical epidural abscesses $[5,6]$, discitis [7], facet joint abscess [8], and meningitis [5] have been reported following spinal injections for treatment of spine pain; however, due to heterogeneity in this specialized subgroup of patients, the incidence of spinal infections following spinal injections remains unknown.

Spinal infections, specifically epidural abscesses, most often occur in patients with impaired immune function or a history of an infectious disease [1]; thus, spinal infections are uncommon in immunocompetent individuals. 
Although spinal infections have been reported following dental procedures [9], development of a spinal infection attributed to poor dentition without a history of a dental procedure in an immunocompetent adult has not been previously reported. Here we provide a case report of a multilevel lumbar spine infection that developed in an immunocompetent adult with poor dentition.

\section{Case presentation}

A 63-year-old white male man with past medical history of hypertension presented to a hospital emergency department with a 4-month history of progressively worsening low back pain. Immediately prior to seeking emergency care, he reported an episode of fever, rigor, and generalized arthralgia. On initial presentation to the emergency department, his heart rate was 84 beats/ minute, blood pressure was $130 / 65$, respiratory rate was 20 breaths/minute, and oral temperature was $38.0{ }^{\circ} \mathrm{C}$. A general examination was significant for a mildly distressed-appearing man and a musculoskeletal examination demonstrated diffuse tenderness in his lumbar spine area to percussive palpation without associated swelling, erythema, or evidence of trauma. The heart, lung, abdominal, and neurological examinations were within normal limits. Laboratory evaluation was significant for a leukocytosis $\left(31,700 \times 10^{9} / \mathrm{L}\right)$ with $91 \%$ neutrophilia, hypokalemia $(2.8 \mathrm{mmol} / \mathrm{L})$, and elevated serum creatinine $(1.6 \mathrm{mg} / \mathrm{dL})$. Four sets of blood cultures were obtained from the antecubital area and rapid influenza A and B immunoassays were negative. Urine analysis was negative for nitrates and leukocyte esterase. A chest X-ray showed no evidence of an infectious process in his lungs. Non-contrast magnetic resonance imaging (MRI) of his lumbar spine demonstrated a prevertebral and presacral fluid collection ventral to the $\mathrm{L} 4$ to L5 and L5 to S1 interspaces (Fig. 1a and b). The abscess extended into his right psoas musculature. There were findings of discitis involving the L5 to S1 interspace with associated edema of the endplates, although no frank destruction of progressive osteomyelitis was identified. Mild T2 hyperintensity at the L4 to L5 interspace in the presence of a ventral abscess and ventral epidural thickening indicated probable discitis involvement at this level. He was admitted to the hospital with a diagnosis of sepsis and piperacillin/tazobactam was intravenously administered. Blood cultures grew pansensitive Streptococcus intermedius in four of four bottles within 45 hours. Interventional radiology services were not available at the hospital and he was referred to our tertiary care institution for further evaluation.

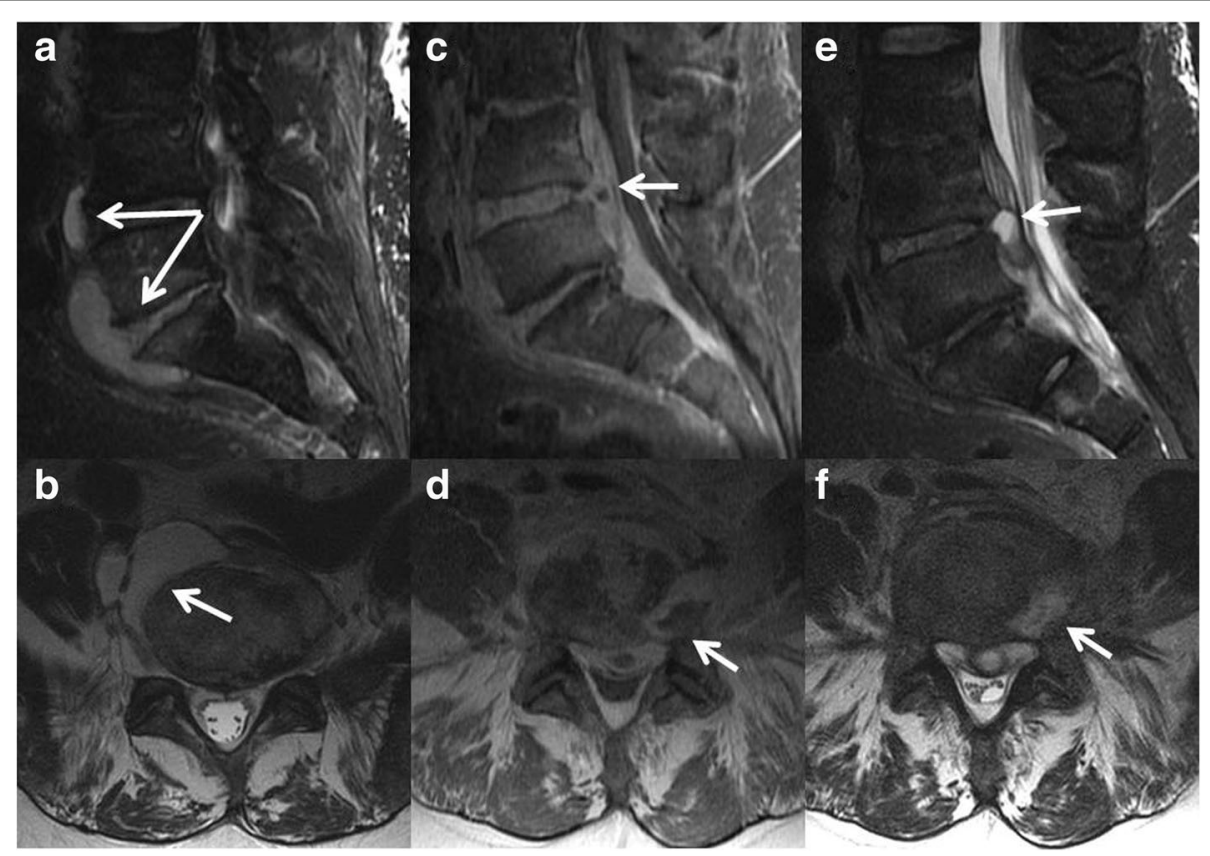

Fig. 1 Initial lumbar spine magnetic resonance imaging performed without gadolinium demonstrated extensive abnormal T2 hyperintensity of the L5 interspace ( $\mathbf{a}$ and $\mathbf{b}$, sagittal fat-saturated T2-weighted image and axial T2-weighted image at the L5 interspace) indicating discitis. A large multilobulated fluid collection was contiguous with the infected interspace indicating an abscess (arrow, $\mathbf{a}$ and $\mathbf{b}$ ). Follow-up lumbar spine magnetic resonance imaging performed with gadolinium demonstrated extensive abnormal enhancement (c and $\mathbf{d}$, post-gadolinium sagittal T1-weighted with fat saturation and axial T1-weighted images, respectively) and T2 hyperintensity (e and $\mathbf{f}$, sagittal T2-weighted with fat saturation and axial T2-weighted at the L4 interspace) involving the $L 3$ to $L 4, L 4$ to $L 5$, and $L 5$ to $S 1$ interspaces. There is abnormal paraspinal and epidural enhancing phlegmon with a focal ventral epidural abscess (arrow, $\mathbf{c}$ and $\mathbf{e}$ ) emanating from the L4 to L5 interspace. An additional abscess involving the left posterolateral soft tissues (arrow, $\mathbf{d}$ and $\mathbf{f}$ ) was targeted during biopsy and yielded $10 \mathrm{cc}$ of fluid 
Despite referral, he elected to delay further diagnostic evaluation and treatment for 2 months. On arrival to our tertiary care center, a lumbar spine MRI demonstrated progression of discitis now involving L3 to L4 through L5 to S1 (Fig. 1c-f). There was marked progression of the ventral epidural phlegmon with new development of a $2 \mathrm{~cm}$ epidural abscess (Fig. 1c and e; arrowhead). There was progression of mild enhancement of the L4 and L5 vertebral bodies indicative of osteomyelitis. An abscess involving the left side of L4 (Fig. 1c and d) had developed. A computed tomography $(\mathrm{CT})$-guided biopsy was performed targeting the erosive abscess and $10 \mathrm{cc}$ of fluid was aspirated. In addition, three core biopsies of the L4 to L5 interspace were taken and subsequent cultures were positive for Streptococcus intermedius. He had no signs of neurological impairment (that is, normal lower extremity strength and sensation) but a physical examination and dental panoramic radiograph demonstrated significant necrotic dentition (Fig. 2). An examination of his oral cavity was negative for masses, lesions, or ulcerations involving the soft tissues, but several carious nonrestorable teeth were observed that were nontender to palpation or percussion and were nonmobile. He reported that his last episode of dental care occurred more than 20 years ago. As a part of treatment, ten teeth were extracted and the necrotic dentition was assumed to be the most likely source of infection. Other testing included a transesophageal echocardiogram which found no evidence of endocarditis. On hospital dismissal, he completed a 12-week course of ceftriaxone as well as extensive dental work to eradicate the source of infection. He remains on orally administered cefadroxil pending repeat imaging.

\section{Discussion}

Commonly occurring signs and symptoms of an epidural abscess include back pain and focal neurological deficits $[1,9]$. However, three aspects of our case report warrant further consideration. First, the organism isolated from L4 to L5 disc space aspirate was a facultative oral bacterium, specifically Streptococcus intermedius. More commonly, the organism isolated from epidural abscesses is Staphylococcus aureus with typical sources arising from the skin or surgical manipulation of the spine $[1,10]$.

Second, our patient did not have any known comorbidities, such as diabetes mellitus, history of an infectious disease, or malignancy that could impair immune function. Moreover, he denied use of illicit intravenously administered drugs and there was no history of spinal surgery; both of which have been reported to be risk factors for epidural abscess $[1,10]$. Rarely have case reports demonstrated epidural abscess formation from a Streptococcal species in an immunocompetent patient and none have reported progression of the infection to involve the disc space and development of osteomyelitis [11, 12].

Third, oral bacteria have been well documented to spread hematogenously following dental procedures [9]. Unique to our case was that the origin of the spinal infection was necrotic dentition, and there was no history of a recent dental procedure or injury to the oral cavity. However, this does not exclude the potential occurrence of dental manipulation by the patient (that is, use of a toothpick). There have been other case reports that link epidural abscesses to a dental origin $[11,12]$, but none reported epidural abscesses that have arisen spontaneously in an immunocompetent patient without any recent history of a dental procedure or injury to the oral cavity.

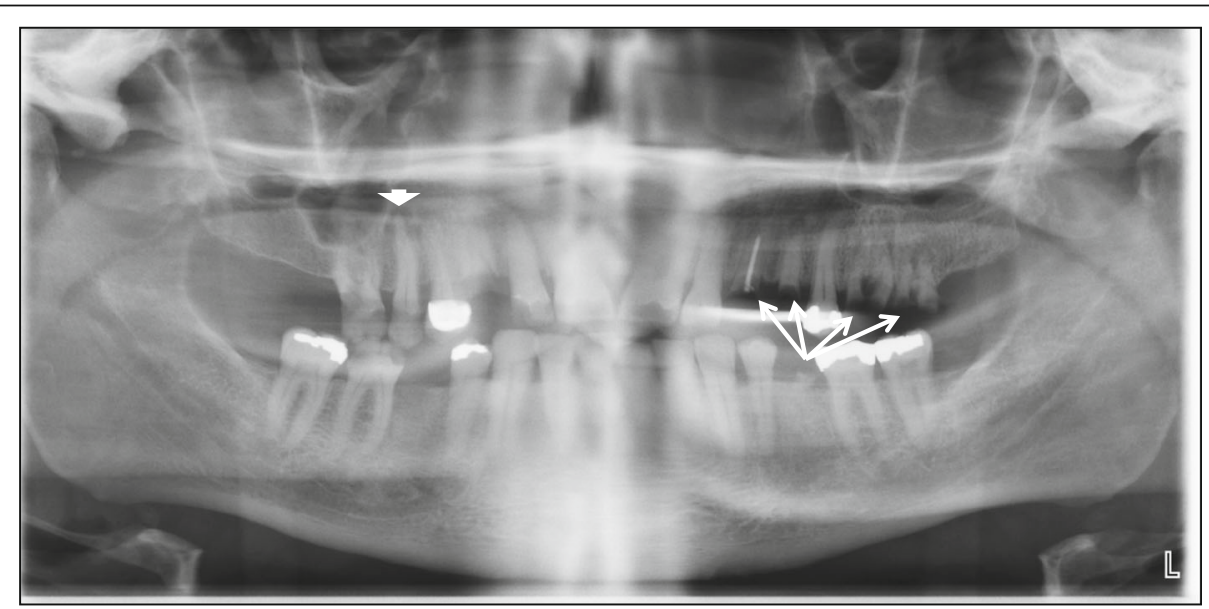

Fig. 2 Dental panoramic radiograph demonstrated multiple missing teeth and dental amalgam due to prior dental caries. Most notably there were numerous erosions of the crowns (arrows) of many teeth with a mottled appearance of the residual root and periapical lucencies (arrowhead). These carious nonrestorable teeth were later extracted 


\section{Conclusions}

This case report demonstrates the importance of determining the source of infection in a patient with a spontaneous spinal infection. Even in the absence of a recent dental procedure, dentition should be considered a possible source of infection in an immunocompetent patient who presents with a spontaneous spinal infection.

\section{Abbreviations}

$\mathrm{CT}$ : Computed tomography; MRI: Magnetic resonance imaging

\section{Acknowledgements}

No applicable

\section{Funding}

No funding was received for this case report

\section{Availability of data and materials}

Data sharing is not applicable to this article because no datasets were generated or analyzed.

\section{Authors' contributions}

CC and WH contributed patient clinical analyses and made substantial contributions to the clinical decision-making processes. MQ, CC, and WM were involved in drafting the manuscript and revising it for intellectual content. All authors read and approved the final manuscript.

\section{Ethics approval and consent to participate}

The Mayo Clinic Institutional Review Board (IRB) acknowledged that the case report did not require IRB review based on submitted responses to an IRB application.

\section{Consent for publication}

Written informed consent was obtained from the patient for publication of this case report and any accompanying images. A copy of the written consent is available for review by the Editor-in-Chief of this journal.

\section{Competing interests}

The authors declare that they have no competing interests.

\section{Publisher's Note}

Springer Nature remains neutral with regard to jurisdictional claims in published maps and institutional affiliations.

\section{Author details}

${ }^{1}$ Department of Anesthesiology, Mayo Clinic Graduate School of Medicine, Rochester, MN, USA. ${ }^{2}$ Department of Radiology, Mayo Clinic College of Medicine, Rochester, MN, USA. ${ }^{3}$ Department of Anesthesiology, Mayo Clinic College of Medicine, 200 First St SW, Rochester, MN 55905, USA.

Received: 20 August 2017 Accepted: 14 October 2017

Published online: 22 November 2017

\section{References}

1. Ptaszynski AE, Hooten WM, Huntoon MA. The incidence of spontaneous epidural abscess in Olmsted County from 1990 through 2000: a rare cause of spinal pain. Pain Med. 2007:8:338-43.

2. Hlavin ML, Kaminski HJ, Ross JS, Ganz E. Spinal epidural abscess: a ten-year perspective. Neurosurgery. 1990;27:177-84.

3. Dahlgren N, Tornebrandt K. Neurological complications after anaesthesia. A follow-up of 18,000 spinal and epidural anaesthetics performed over three years. Acta Anaesthesiol Scand. 1995;39:872-80.

4. Wang LP, Hauerberg J, Schmidt JF. Incidence of spinal epidural abscess after epidural analgesia: a national 1-year survey. Anesthesiology. 1999;91:1928-36.

5. Hooten WM, Kinney MO, Huntoon MA. Epidural abscess and meningitis after epidural corticosteroid injection. Mayo Clin Proc. 2004;79:682-6.

6. Koka VK, Potti A. Spinal epidural abscess after corticosteroid injections. South Med J. 2002;95:772-4.
7. Hooten WM, Mizerak A, Carns PE, Huntoon MA. Discitis after lumbar epidural corticosteroid injection: a case report and analysis of the case report literature. Pain Med. 2006;7:46-51.

8. Hoelzer BC, Weingarten TN, Hooten WM, Wright RS, Wilson WR, Wilson PR. Paraspinal abscess complicated by endocarditis following a facet joint injection. Eur J Pain. 2008;12:261-5.

9. Muzii VF, Mariottini A, Zalaffi A, Carangelo BR, Palma L. Cervical spine epidural abscess: experience with microsurgical treatment in eight cases. J Neurosurg Spine. 2006;5:392-7.

10. Lieber B, Han B, Strom RG, Mullin J, Frempong-Boadu AK, Agarwal N, Kazemi N, Tabbosha M. Preoperative Predictors of Spinal Infection within the National Surgical Quality Inpatient Database. World Neurosurg. 2016;89:517-24.

11. Ramhmdani S, Bydon A. Streptococcus intermedius: an unusual cause of spinal epidural abscess. J Spine Surg. 2017;3:243-9.

12. Kaye ID, Protopsaltis TS. Cervical Facet Joint Infection and Associated Epidural Abscess with Streptococcus intermedius from a Dental Infection Origin A Case Report and Review. Bull Hosp Jt Dis (2013). 2016;74:237-43.
Submit your next manuscript to BioMed Central and we will help you at every step:

- We accept pre-submission inquiries

- Our selector tool helps you to find the most relevant journal

- We provide round the clock customer support

- Convenient online submission

- Thorough peer review

- Inclusion in PubMed and all major indexing services

- Maximum visibility for your research

Submit your manuscript at www.biomedcentral.com/submit
C Biomed Central 